\title{
Iodine(III)-Mediated Ring Contraction Reactions: Synthesis of Oxygen- and Nitrogen-Substituted Indanes
}

\author{
Anees Ahmad and Luiz F. Silva Júnior* \\ Instituto de Química, Universidade de São Paulo, CP 26077, 05513-970 São Paulo-SP, Brazil
}

\begin{abstract}
The synthesis of oxygen- and nitrogen-substituted indanes was successfully performed by iodine(III)-mediated ring contraction of 1,2-dihydronaphthalenes. Acetoxy and benzoyloxy alkenes afforded indanes in 60-71\% yield, irrespective of their position on aromatic ring. Similarly, the nitrogen containing substrates protected with 9-fluorenylmethyloxycarbonyl (Fmoc) and benzoyl (Bz) groups smoothly undergoes ring contraction giving indanes in $64-77 \%$ yield. The tosyl-protected substrate resulted only in addition products.
\end{abstract}

Keywords: indane, hypervalent iodine, ring contraction, 1,2-dihydronaphthalenes

\section{Introduction}

Indane skeleton occurs in many biologically active natural products ${ }^{1,2}$ and pharmaceuticals, constituting an important target in organic synthesis and in drug discovery. ${ }^{3}$ Numerous synthetic methodologies have been developed to construct indane core including cyclization, ${ }^{4-6}$ cycloaddition, ${ }^{7-9}$ and rearrangements. ${ }^{10-13}$ Indanes substituted in the aromatic ring by nitrogen ${ }^{14-18}$ or by oxygen ${ }^{2,19-22}$ are present in compounds with promising biological activities. Hypervalent iodine reagents play a substantial role in chemical synthesis, promoting efficiently carbon-carbon bond formations, rearrangements and functional group interconversions, including asymmetric versions for many reactions. ${ }^{23-28}$ The iodine(III)-mediated ring contraction of 1,2-dihydronaphthalenes gives functionalized indanes (Scheme 1, 1a). ${ }^{13,29}$ This transformation was successfully employed in total syntheses, such as (+)-mutisianthol, ${ }^{30}$ ( \pm )-indatraline,,${ }^{29}$ and $(+)$-trans-trikentrin A. ${ }^{31}$ Although the reactivity of several substrates was investigated under many conditions, the tolerance for substitution at aromatic ring was not high. For example, methoxy-substituted substrates furnished the desired indanes in low yield and additionally the main product were those related to the addition of solvent, as exemplified by the behavior of compound $\mathbf{1 b}$. Motivated by the importance of oxygen- and nitrogensubstituted indanes, we herein show the ring contraction of additional 1,2-dihydronaphthalenes with iodine(III).

*e-mail: luizfsjr@iq.usp.br

\section{Results and Discussion}

The preparation of the required substrates was performed as described in the following paragraphs. The protected amine tetralones (7c-f) were prepared by classical transformations (Table 1). Amine tetralone 5 in the presence of benzoyl chloride $(\mathrm{BzCl})$, triethylamine $\left(\mathrm{Et}_{3} \mathrm{~N}\right)$ and dichloromethane (DCM) as solvent gave benzoyl protected amine $\mathbf{7 c}$ in $89 \%$ yield (entry 1 ). ${ }^{32}$ The tosyl protected amine tetralone 7d was obtained in $96 \%$ yield in pyridine with tosyl chloride (p-TsCl) (entry 2). ${ }^{33}$ In a similar manner, base labile Fmoc protected amine $\mathbf{7 e}$ was formed in $98 \%$ yield with 9-fluorenylmethyloxycarbonyl chloride (Fmoc-Cl) and pyridine in DCM (entry 3). ${ }^{34}$ The acetylation of tetralone 6 was achieved using 4-dimethylaminopyridine (DMAP), acetic anhydride $\left(\mathrm{Ac}_{2} \mathrm{O}\right)$ in $\mathrm{Et}_{3} \mathrm{~N}$ giving acetyl protected ketone $7 \mathbf{f}$ in $97 \%$ yield (entry 4$){ }^{13,35}$

Several cyclic olefinic substrates were synthesized via reduction/dehydration protocol. The commercially available hydroxy ketone $\mathbf{8 g}$ was reduced with $\mathrm{NaBH}_{4}$ in $\mathrm{MeOH}$ to the corresponding alcohol as a white solid. Dehydration of alcohol at $130{ }^{\circ} \mathrm{C}$ in the presence of phosphoric acid $\left(\mathrm{H}_{3} \mathrm{PO}_{4}\right)$ gave alkene product $1 \mathrm{~g}$ in $76 \%$ yield (Table 2, entry 1). ${ }^{35}$ The methoxy functionalized ketones $\mathbf{8 h}$ and $\mathbf{8 b}$ were also transformed into cyclic alkenes $\mathbf{1 h}$ and $\mathbf{1 b}$ in 93 and $76 \%$ yields, respectively (entries 2 and 3)..$^{13,36,37}$ The benzoyl, tosyl, Fmoc and acetyl protected amino ketones (7c-f) were smoothly converted into desired olefinic substrates (1c-f) in good yields (entries 4-7). ${ }^{13,38}$ 


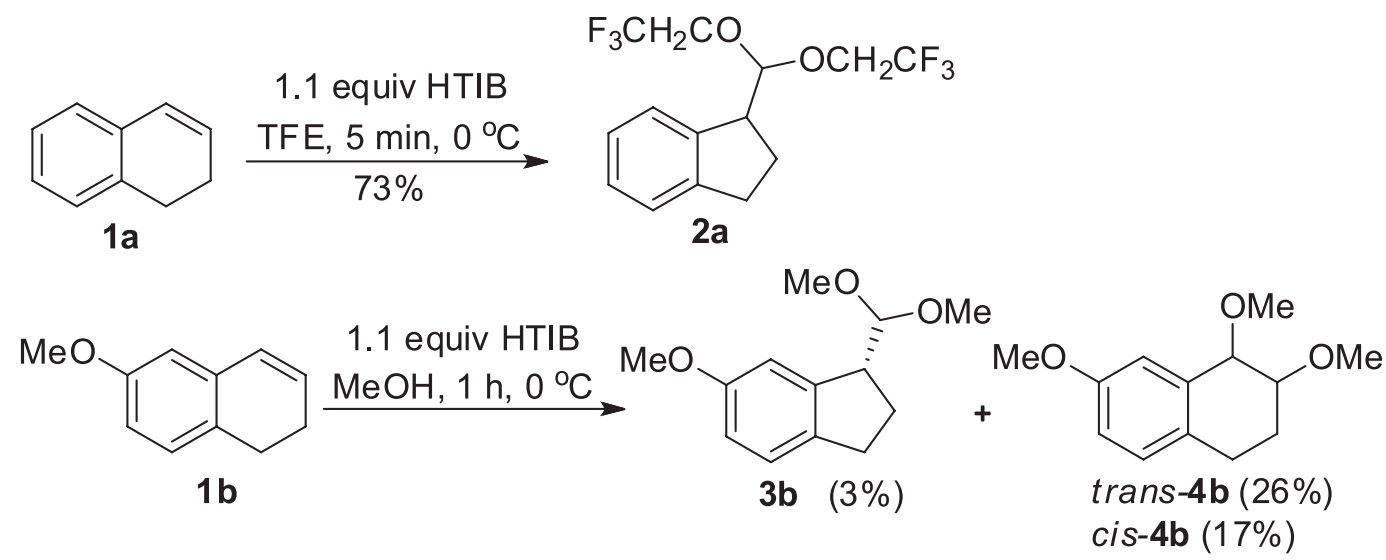

Scheme 1. Oxidation of 1,2-dihydronaphthalenes 1a-b with iodine(III).

Table 1. Synthesis of protected amines tetralones 7c-f

\begin{tabular}{|c|c|c|c|}
\hline entry & Substrate & Condition & Product (Yield) \\
\hline 1 & & $\begin{array}{c}\mathrm{BzCl}, \mathrm{Et}_{3} \mathrm{~N} \\
\mathrm{DCM}, \mathrm{rt}, 3 \mathrm{~h}\end{array}$ & \\
\hline 2 & & $\begin{array}{l}p \mathrm{TsCl} \text { pyridine } \\
\text { reflux, } 16 \mathrm{~h}, \mathrm{rt}\end{array}$ & \\
\hline 3 & & $\begin{array}{c}\text { Fmoc-Cl pyridine } \\
\text { DCM, } 0^{\circ} \mathrm{C} \text { to } \mathrm{rt}, 1 \mathrm{~h}\end{array}$ & \\
\hline 4 & & $\begin{array}{c}\mathrm{Ac}_{2} \mathrm{O} \\
\text { DMAP Et }{ }_{3} \mathrm{~N}, \mathrm{rt}, 1 \mathrm{~h}\end{array}$ & \\
\hline
\end{tabular}

DCM: dichloromethane; rt: room temperature; DMAP: 4-dimethylaminopyridine.

The acetylation of phenol $\mathbf{1 g}$ was accomplished with DMAP, $\mathrm{Ac}_{2} \mathrm{O}$ in $\mathrm{Et}_{3} \mathrm{~N}$ as solvent, ${ }^{13}$ leading to protected alkene $9 \mathrm{~g}$ in $96 \%$ yields (Table 3, entry 1). Similarly, phenol $1 \mathrm{~g}$ was also protected with benzoyl group in $94 \%$ yield using $\mathrm{BzCl}$ and $\mathrm{Et}_{3} \mathrm{~N}$ as a base (entry 2). ${ }^{32}$ Methyl ethers ( $\mathbf{1 h}$ and $\mathbf{1 b})$ were first demethylated using sodium ethanethiolate (generated in situ) in dimethylformamide (DMF) at $140{ }^{\circ} \mathrm{C}$ giving the corresponding phenol, ${ }^{39,40}$ which were acetylated under standard conditions (entries 3 and 4).
Iodine(III) is known to act as single-electron-transfer (SET) for methoxy-substituted aromatic compounds forming reactive cation radical intermediates. ${ }^{41,42}$ The generation of these cation radicals could be a possible reason of rearrangement failure in substrates like $\mathbf{1 b}$, due to their susceptibility towards nucleophilic attack and other side reactions. Thus, we consider that the ring contraction could take place with an acetyl group instead of a methoxy making it useful in the synthesis of oxygen-substituted indanes. Several reaction conditions were tested for the 
Table 2. Preparation of dihydronaphthalenes $\mathbf{1 b}$-h via reduction/dehydration

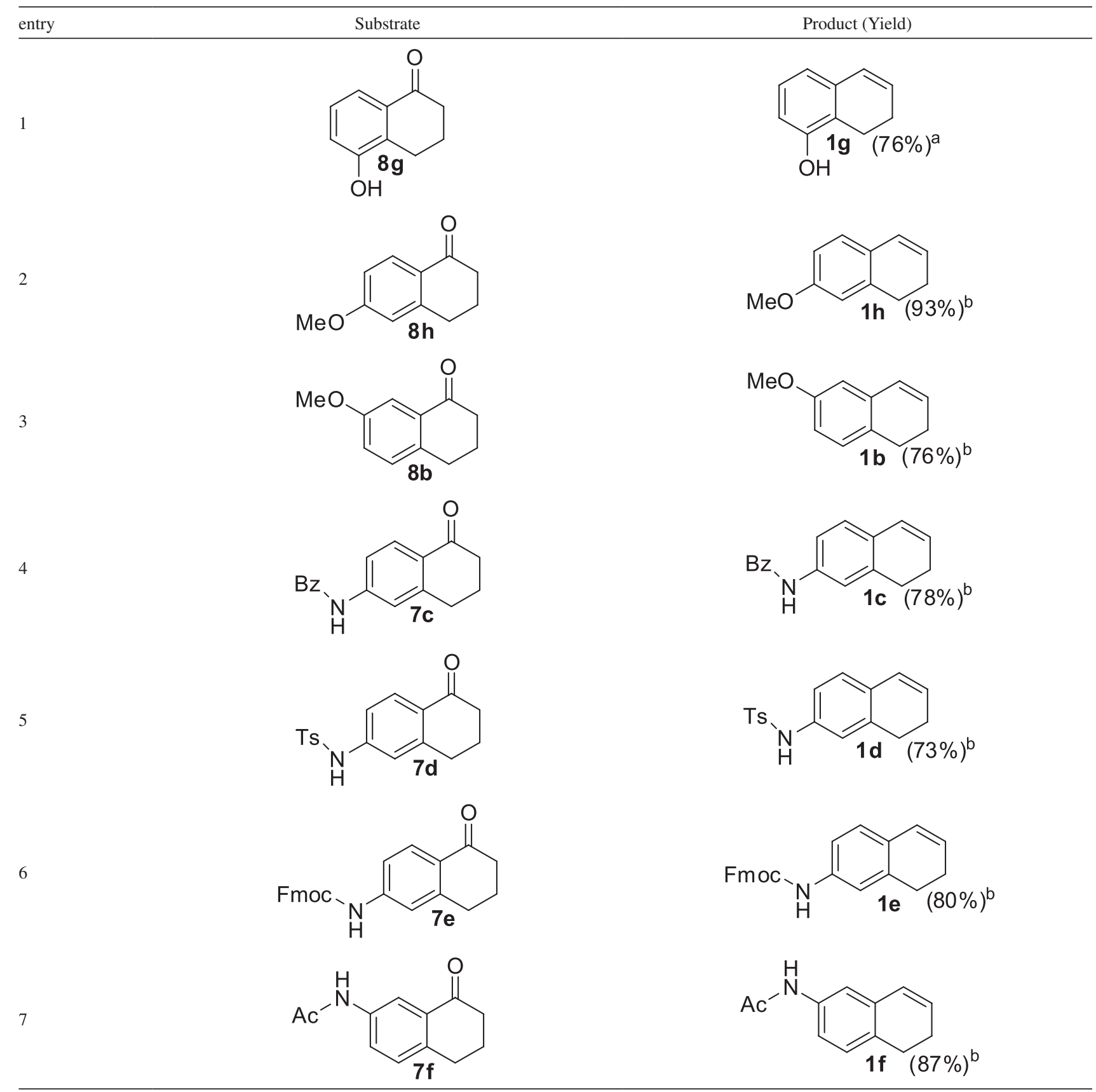

${ }^{\mathrm{a}}($ i $) \mathrm{NaBH}_{4}, \mathrm{MeOH}, 0{ }^{\circ} \mathrm{C}$ to rt, $1 \mathrm{~h}$; (ii) $\mathrm{H}_{3} \mathrm{PO}_{4}, \mathrm{THF}, 80^{\circ} \mathrm{C}, 2 \mathrm{~h} ;{ }^{\mathrm{b}}($ i $) \mathrm{NaBH}_{4}, \mathrm{MeOH}, 0{ }^{\circ} \mathrm{C}$ to rt, $1 \mathrm{~h}$; (ii) cat. TsOH.H $\mathrm{H}_{2} \mathrm{O}$, toluene, $130{ }^{\circ} \mathrm{C}, 2 \mathrm{~h}$.

oxidation of alkene $9 \mathrm{~g}$ with $\mathrm{PhI}(\mathrm{OH}) \mathrm{OTs}$ (HTIB) (Table 4). The desired ring contraction product $2 \mathrm{~g}$ was successfully obtained in $47 \%$ yield using trifluoroethanol (TFE)/DCM (1:4) as solvent (entry 1). Using pure TFE, the yield of acetal $2 \mathrm{~g}$ increased to $66 \%$ (entry 2). The deprotection of substrate $9 \mathrm{~g}$ into phenol $\mathbf{1 g}$ was observed when $\mathrm{MeOH}$ was used as solvent (entry 3 ).

With the optimized conditions, ring contraction was successfully carried out in other oxygenated substrates
(Table 5). Benzoyl protected alkene 9gg gave acetal 2 gg in $71 \%$ yield (entry 1). Acetyl protected alkene $\mathbf{9 h}$ smoothly afforded product $2 \mathrm{~h}$ in $60 \%$ yield (entry 2). Acetyl alkene $\mathbf{9 b}$ gave acetal $\mathbf{2 b}$ in $65 \%$ yield (entry 3 ).

Oxidation with HTIB was also studied in amine protected alkenes (Table 6). Benzoyl protected alkene 1c experienced ring contraction giving acetal product $\mathbf{3 c}$ in $77 \%$ yield in $\mathrm{MeOH} / \mathrm{DCM}(8: 1)$ as solvent (entry 1). DCM was added to solubilize substrate $1 \mathbf{c}$ and to increase 
Table 3. Preparation of O-substituted dihydronaphthalenes

entry

DMAP: 4-dimethylaminopyridine; DCM: dichloromethane; DMF: dimethylformamide.

Table 4. Reactions of acetyl protected alkene $9 \mathrm{~g}$ with HTIB

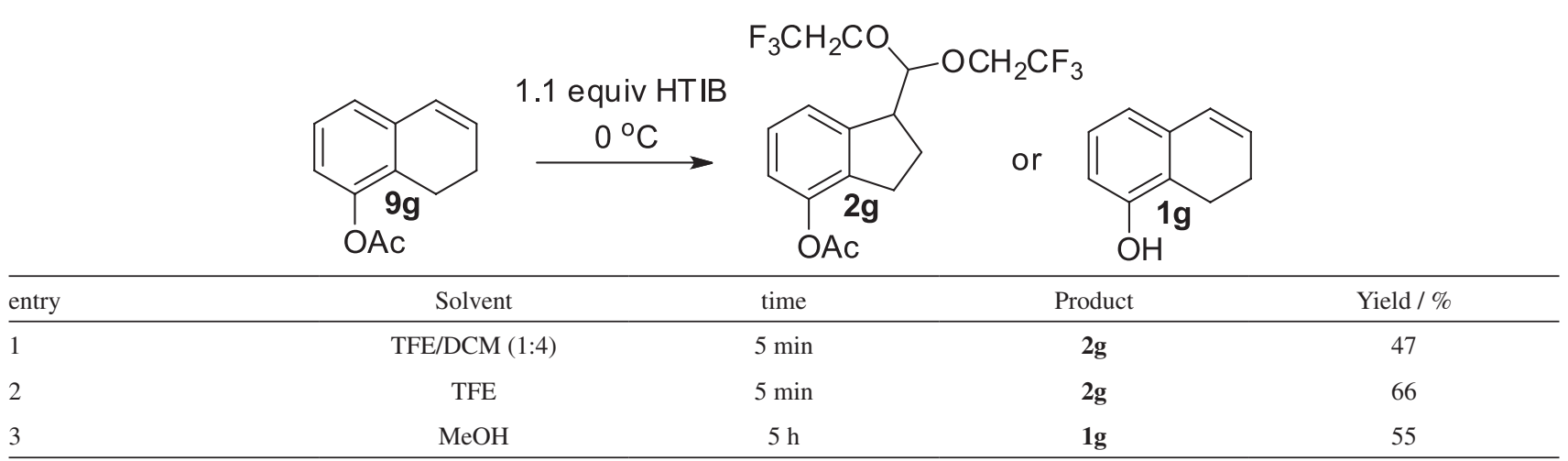

TFE: trifluoroethanol; DCM: dichloromethane.

the rate of reaction. When the reaction was tried in pure $\mathrm{MeOH}$, indane $3 \mathbf{c}$ was obtained in $51 \%$ yield and took $1 \mathrm{~h}$ to consume all starting material (entry 2). The protecting group tolerance was further investigated with Fmoc protected alkene 1e and the anticipated ring contraction product $3 \mathrm{e}$ was isolated in $64 \%$ yield (entry 3 ).

The reaction of tosyl protected 7-amine alkene 1d with HTIB was also investigated under different reaction conditions (Table 7). Tosyl amide could have a facilitating effect on ring contraction by increasing electronic density on migrating carbon $\mathbf{4 a} .{ }^{13}$ However, only the formation of addition products trans-4d and cis-4d were observed in $\mathrm{MeOH}$ and in $\mathrm{DCM} / \mathrm{MeOH}$ as solvents (entries 1 and 2). Fluorinated solvents like TFE and hexafluoroisopropanol
(HFIP) proved to be ineffective under the conditions tested (entries 3 and 4).

Oxidation in acetyl protected 6-amine alkene 1f was explored. In this case, amide substituent meta to migrating carbon would decrease the electronic density by inductive effect of nitrogen atom and does not increase directly the electronic density at migrating carbon by mesomeric effect. ${ }^{13}$ Several conditions were tested for ring contraction of alkene $\mathbf{1 f}$ (Table 8). Slow reaction and complex mixtures were observed for substrate 1f using HTIB either in $\mathrm{MeOH}$ or in $\mathrm{DCM} / \mathrm{MeOH}$ at different temperatures (entries 1-3). Fluorinated solvents, such as TFE and HFIP/DCM, also did not provide ring contraction product (entries 4 and 5). 
Table 5. Reactions of oxygenated alkenes $\mathbf{9 b}, \mathbf{9 g g}$ and $\mathbf{9 h}$ with 1.2 equiv of HTIB

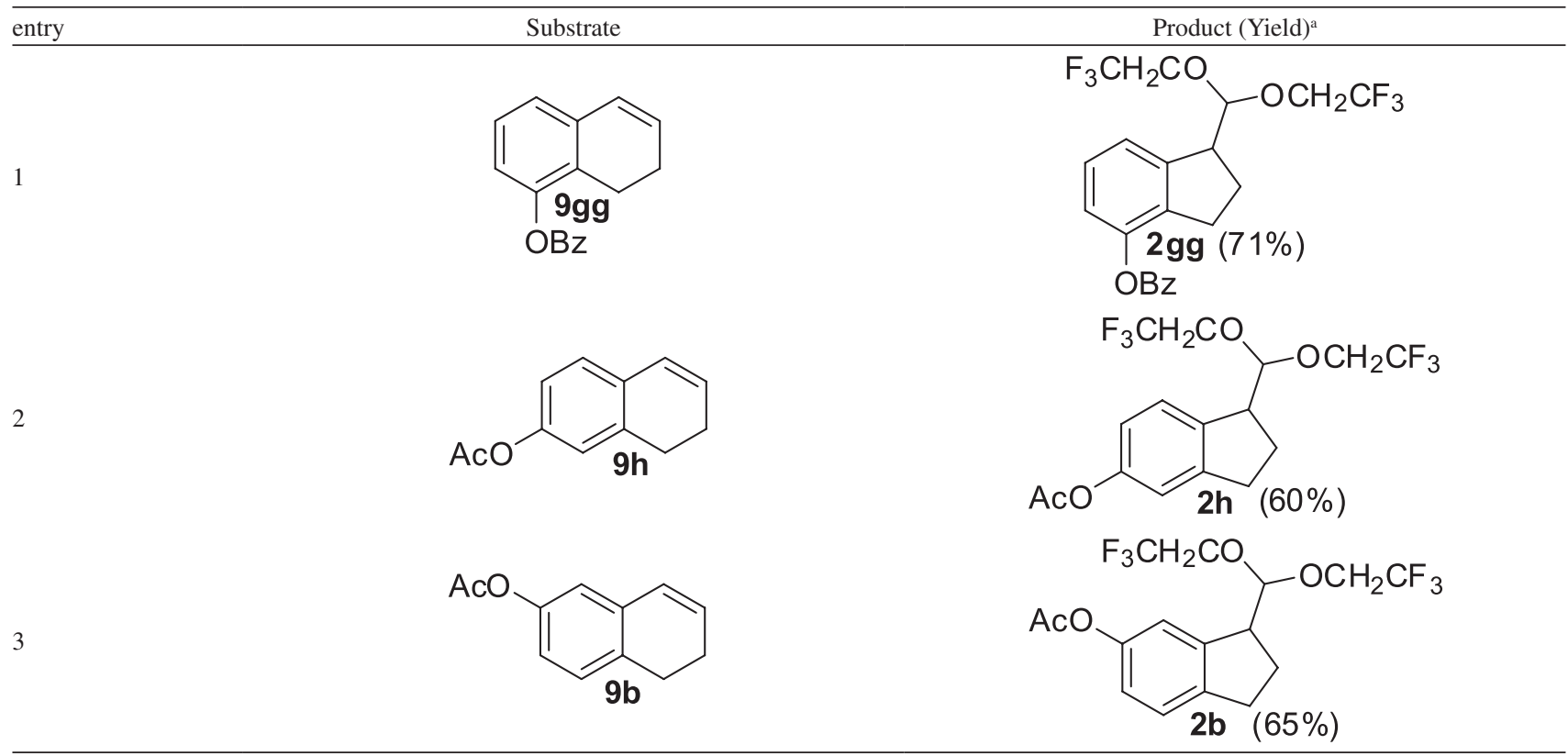

Trifluoroethanol, $0{ }^{\circ} \mathrm{C}, 5 \mathrm{~min}$.

Table 6. Reaction of 7 -amine alkenes with 1.2 equiv HTIB at $0{ }^{\circ} \mathrm{C}$

entry

DCM: dichloromethane.

\section{Conclusions}

In conclusion, the ring contraction of 1,2-dihydronaphthalenes using HTIB was expanded to substrates bearing oxygen and nitrogen substituents in the aromatic ring. Oxidative rearrangement was successfully carried out in oxygenated substrates independent on their position on aromatic ring. Acetoxy and benzoyloxy alkenes afforded indanes in $60-71 \%$ yield. The $N$-protecting groups Fmoc and $\mathrm{Bz}$ are stable under the reaction conditions giving indanes in $64-77 \%$ yield. The Ts-protected substrate gave only addition products. The results showed the tolerance 
Table 7. Reaction of tosylamide 1d with HTIB

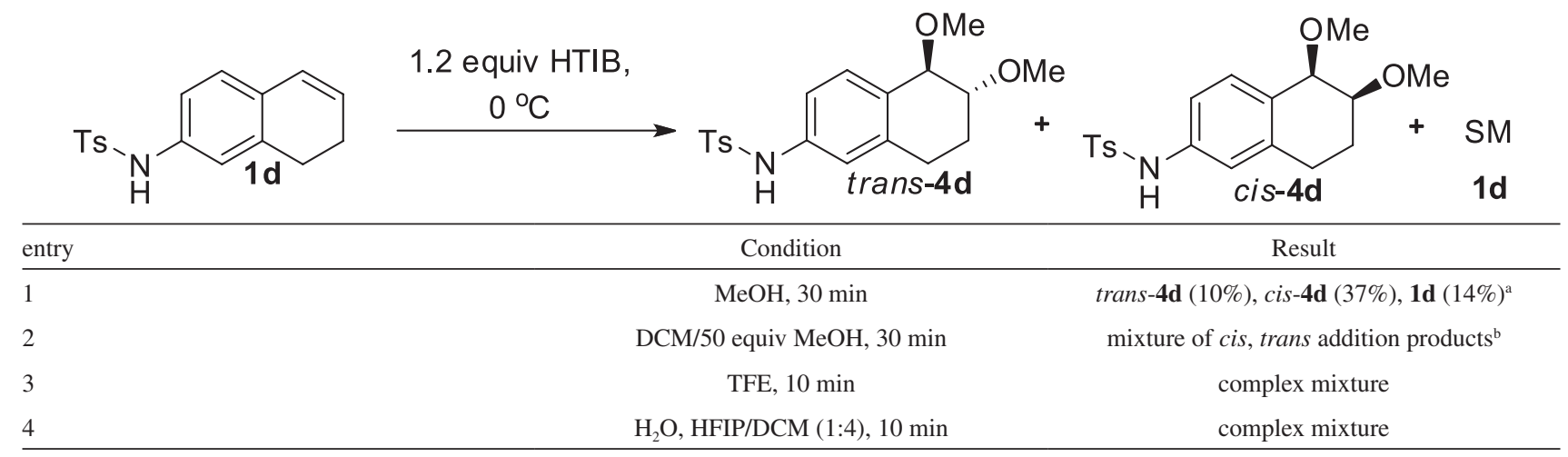

asolated yield; ' $\mathrm{GC}$ monitored; DCM: dichloromethane; HFIP: hexafluoroisopropanol.

Table 8. Reaction of 6-acetamide $\mathbf{1 f}$ with HTIB

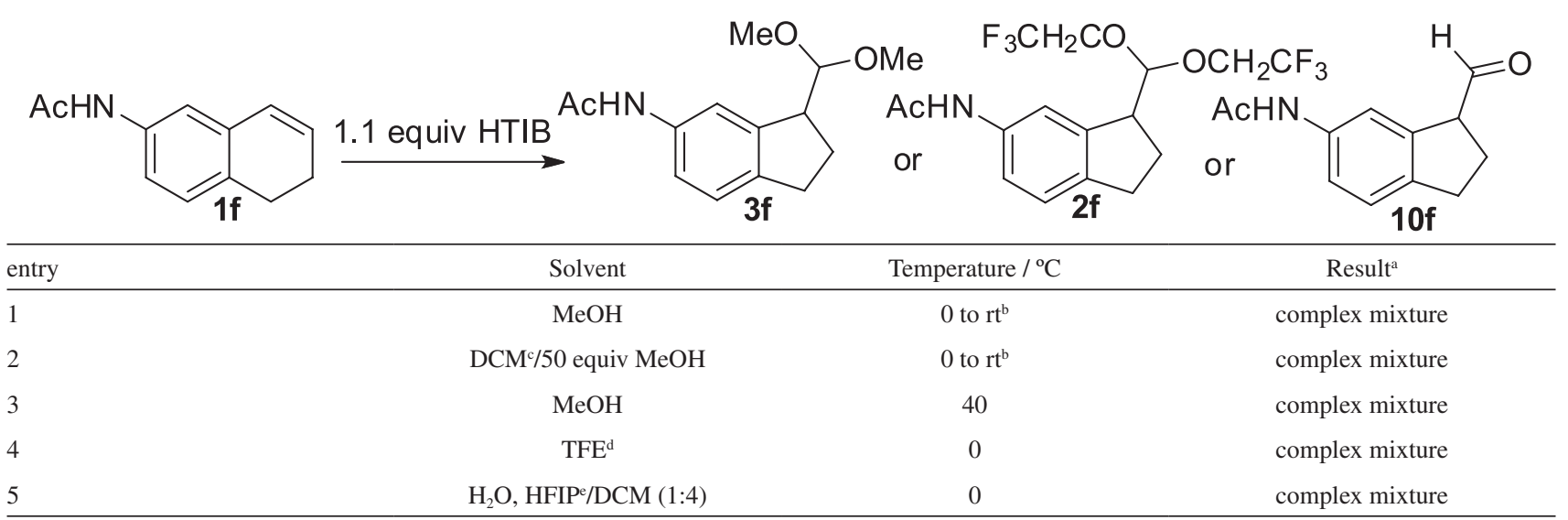

${ }^{a}$ By TLC and GC; ${ }^{b}$ rt: room temperature; ${ }^{c D C M}$ : dichloromethane; ${ }^{\mathrm{d} T F E}$ : trifluoroethanol; ${ }^{\mathrm{e}} \mathrm{HFIP}$ : hexafluoroisopropanol.

of protecting groups in ring contraction reaction mediated by HTIB.

\section{Experimental}

All commercially available reagents were used without further purification unless otherwise noted. All solvents used for reactions and chromatography were dried and purified by standard methods. ${ }^{43}$ Thin-layer chromatography (TLC) analyses were performed using silica gel 60F 254 precoated plates, with detection by UV-absorption $(254 \mathrm{~nm})$ and by spraying with $p$-anisaldehyde and phosphomolybdic acid solutions followed by charring at ca. $150{ }^{\circ} \mathrm{C}$ for visualization. Flash column chromatography was performed using silica gel 200-400 Mesh. All nuclear magnetic resonance (NMR) analyses were recorded using $\mathrm{CDCl}_{3}$ as solvent and tetramethylsilane (TMS) as internal standard. Chemical shifts are reported in ppm downfield from TMS with reference to internal solvent.

\section{Preparation of substrates}

\section{$\mathrm{N}$-(7,8-Dihydronaphthalen-2-yl)benzamide (1c)}

To a stirred solution of ketone 5 ( $0.677 \mathrm{~g}, 4.20 \mathrm{mmol})$ and $\mathrm{Et}_{3} \mathrm{~N}(0.65 \mathrm{~mL}, 0.467 \mathrm{~g}, 4.62 \mathrm{mmol})$ in DCM $(35 \mathrm{~mL})$ was added $\mathrm{BzCl}(0.683 \mathrm{~mL}, 0.826 \mathrm{~g}, 5.88 \mathrm{mmol})$. After $3 \mathrm{~h}$, the reaction mixture was washed with $10 \% \mathrm{HCl}$, saturated solution of $\mathrm{NaHCO}_{3}$ and dried over anhydrous $\mathrm{MgSO}_{4}$. Purification by flash column chromatography (40-60\% EtOAc in hexane) gave benzoyl protected ketone 7c. ${ }^{44}$ Yield: $0.995 \mathrm{~g}(89 \%)$; light yellow solid; mp: 193.5-195.2 ${ }^{\circ} \mathrm{C} ;{ }^{1} \mathrm{H} \mathrm{NMR}\left(300 \mathrm{MHz}, \mathrm{CDCl}_{3}\right) \delta 2.13$ (quin, $2 \mathrm{H}, J 6.3 \mathrm{~Hz}), 2.64(\mathrm{t}, 2 \mathrm{H}, J 6.3 \mathrm{~Hz}), 2.97(\mathrm{t}, 2 \mathrm{H}, J 6 \mathrm{~Hz})$, 7.37 (dd, 1H, J 8.4, 1.8 Hz), 7.45-7.52 (m, 2H), 7.55-7.61 (m, 1H), 7.86-7.89 (m, 3H), 8.04 (d, 1H, J $8.7 \mathrm{~Hz}), 8.11$ $(\mathrm{d}, 1 \mathrm{H}, J 6.9 \mathrm{~Hz}) ;{ }^{13} \mathrm{C} \mathrm{NMR}\left(75 \mathrm{MHz}, \mathrm{CDCl}_{3}\right) \delta 23.2,30.0$, $117.8,118.8,127.1,128.6,128.8,130.1,132.2,134.4$, $142.3,146.3,165.8,197.4$. To a two neck round bottom flask with magnetic stirrer under nitrogen atmosphere was added $\mathrm{NaBH}_{4}(0.170 \mathrm{~g}, 4.50 \mathrm{mmol})$ to the solution of 
ketone 7c $(0.995 \mathrm{~g}, 3.75 \mathrm{mmol})$ in $\mathrm{MeOH}(40 \mathrm{~mL})$. The mixture was stirred for 30 minutes at $0{ }^{\circ} \mathrm{C}$. The temperature was raised to room temperature (rt) for another $1 \mathrm{~h}$. The reaction was quenched by addition of distilled $\mathrm{H}_{2} \mathrm{O}(10 \mathrm{~mL})$ and the mixture was extracted with EtOAc $(3 \times 30 \mathrm{~mL})$. The combined organic extracts were washed with brine $(2 \times 10 \mathrm{~mL})$ and dried over anhydrous $\mathrm{MgSO}_{4}$. The solvent was removed under reduced pressure and $N$-(5-hydroxy5,6,7,8-tetrahydronaphthalen-2-yl)benzamide was obtained as white solid $(0.980 \mathrm{~g}, 3.67 \mathrm{mmol}, 98.0 \%)$ and used in the next step without purification. The $N$-(5-hydroxy5,6,7,8-tetrahydronaphthalen-2-yl)benzamide $(0.980 \mathrm{~g}$, $3.67 \mathrm{mmol})$ was dissolved in toluene $(30 \mathrm{~mL})$ with the addition of few crystals of $\mathrm{TsOH} . \mathrm{H}_{2} \mathrm{O}$ in round bottom flask, fitted with Dean-Stark apparatus. The system was maintained at $130{ }^{\circ} \mathrm{C}$ and the reaction was monitored by TLC. The reaction was quenched by addition of saturated solution of $\mathrm{NaHCO}_{3}$ and extracted with hexane $(3 \times 30 \mathrm{~mL})$. The combined organic extracts were washed with brine $(2 \times 10 \mathrm{~mL})$ and dried over anhydrous $\mathrm{MgSO}_{4}$. The solvent was removed at reduced pressure and the residue was purified by flash chromatography $(15-20 \%$ EtOAc in hexane) giving compound 1c. ${ }^{38}$ Yield: $0.735 \mathrm{~g}$ (80\%); white solid; mp: $170.2-172{ }^{\circ} \mathrm{C} ;{ }^{1} \mathrm{H} \mathrm{NMR}(300 \mathrm{MHz}$, $\left.\mathrm{CDCl}_{3}\right) \delta 2.30-2.36(\mathrm{~m}, 2 \mathrm{H}), 2.83(\mathrm{t}, 2 \mathrm{H}, J 8.2 \mathrm{~Hz}), 5.99$ $(\mathrm{dt}, 1 \mathrm{H}, J 9.2,4.5 \mathrm{~Hz}), 6.45(\mathrm{dt}, 1 \mathrm{H}, J 9.6,1.8 \mathrm{~Hz}), 7.02(\mathrm{~d}$, $1 \mathrm{H}, J 8.1 \mathrm{~Hz}), 7.35$ (dd, $1 \mathrm{H}, J$ 8.1, 2.4 Hz), 7.46-7.58 (m, 4H), 7.75 (bs, 1H), 7.84-7.88 (m, 2H); ${ }^{13} \mathrm{C}$ NMR (75 MHz, $\left.\mathrm{CDCl}_{3}\right) \delta 23.0,27.7,117.8,119.4,126.3,126.9,127.1$, $127.9,128.8,130.8,131.8,135.1,136.4,136.6,165.4$.

$N$-(7,8-Dihydronaphthalen-2-yl)-4-methylbenzenesulfonamide (1d)

To a stirred solution of ketone $5(0.161 \mathrm{~g}, 1.00 \mathrm{mmol})$ in pyridine $(5 \mathrm{~mL})$ was added $\mathrm{TsCl}(0.191 \mathrm{~g}, 1.35 \mathrm{mmol})$. The reaction mixture was heated at reflux for $16 \mathrm{~h}$. The solvent was removed under reduced pressure and the crude residue was extracted with EtOAc $(3 \times 10 \mathrm{~mL})$ and washed with $1 \mathrm{~mol} \mathrm{~L}^{-1} \mathrm{HCl}(10 \mathrm{~mL})$, water $(10 \mathrm{~mL})$ and brine $(10 \mathrm{~mL})$ and dried over anhydrous $\mathrm{MgSO}_{4}$. Purification by flash column chromatography (40-50\% EtOAc in hexane) gave tosyl protected ketone 7d. ${ }^{45}$ Yield: $0.304 \mathrm{~g}$ (96\%); white solid; mp: 214.2-215.6 ${ }^{\circ} \mathrm{C}$; ${ }^{1} \mathrm{H}$ NMR $\left(300 \mathrm{MHz}, \mathrm{CDCl}_{3}\right.$ ) $\delta 2.03(\mathrm{~m}, 2 \mathrm{H}), 2.39(\mathrm{~s}, 3 \mathrm{H}), 2.59(\mathrm{t}, 2 \mathrm{H}, J 6.4 \mathrm{~Hz}), 2.87(\mathrm{t}$, $2 \mathrm{H}, J 6 \mathrm{~Hz}), 6.94(\mathrm{dd}, 1 \mathrm{H}, J 8.7,2.4 \mathrm{~Hz}), 6.98-6.99(\mathrm{~m}, 1 \mathrm{H})$, 7.15 (bs, 1H), 7.25-7.33 (m, 2H), $7.74(\mathrm{~d}, 1 \mathrm{H}, J 8.1 \mathrm{~Hz}), 7.90$ $(\mathrm{d}, 1 \mathrm{H}, J 8.4 \mathrm{~Hz}) ;{ }^{13} \mathrm{C} \mathrm{NMR}\left(75 \mathrm{MHz}, \mathrm{CDCl}_{3}\right) \delta 21.6,23.1$, $29.8,38.8,117.2,118.1,124.8,127.2,129.1,129.9,135.9$, 141.1, 144.4, 146.3, 197.1. The reaction was performed using ketone 7d $(0.788 \mathrm{~g}, 2.50 \mathrm{mmol}), \mathrm{MeOH}(30 \mathrm{~mL})$ and $\mathrm{NaBH}_{4}(0.283 \mathrm{~g}, 7.50 \mathrm{mmol})$. After workup, solvent was removed under reduced pressure and $N$-(5-hydroxy-5,6,7,8tetrahydronaphthalen-2-yl)-4-methylbenzenesulfonamide (0.761 g, $2.39 \mathrm{mmol}, 96.0 \%)$ was obtained as yellowish white solid and used in the next step without purification. The reaction was performed using $N$-(5-hydroxy-5,6,7,8tetrahydronaphthalen-2-yl)-4-methylbenzenesulfonamide (0.761 g, $2.39 \mathrm{mmol})$, toluene $(30 \mathrm{~mL})$ and $\mathrm{TsOH} . \mathrm{H}_{2} \mathrm{O}$ (cat. few crystals) at $130{ }^{\circ} \mathrm{C}$. The crude product was purified by flash column chromatography (15-20\% EtOAc in hexane) giving alkene 1d. Yield: $0.542 \mathrm{~g}$ (76\%); white solid; mp: 126.7-127.8 ${ }^{\circ} \mathrm{C}$; IR (film) $v_{\max } / \mathrm{cm}^{-1} 3524,3257,3033$, 2930, 2883, 2830, 1916, 1735, 1609, 1599, 1575, 1497, $1465,1397,1341,1329,1314,1291,1241,1209,1185$, 1163, 1124; ${ }^{1} \mathrm{H}$ NMR $\left(300 \mathrm{MHz}, \mathrm{CDCl}_{3}\right) \delta$ 2.21-2.29 (m, 2H), 2.37 (s, 3H), 2.69 (t, 2H, J 8.4 Hz), $5.96(\mathrm{dt}, 1 \mathrm{H}$, $J$ 9.6, $4.4 \mathrm{~Hz}), 6.36$ (dt, $1 \mathrm{H}, J 9.6 \mathrm{~Hz}), 6.78-6.86(\mathrm{~m}, 4 \mathrm{H})$, $7.22(\mathrm{~d}, 2 \mathrm{H}, J 8.1 \mathrm{~Hz}), 7.67(\mathrm{~d}, 2 \mathrm{H}, J 8.4 \mathrm{~Hz}) ;{ }^{13} \mathrm{C} \mathrm{NMR}$ $\left(75 \mathrm{MHz}, \mathrm{CDCl}_{3}\right) \delta 21.5\left(\mathrm{CH}_{3}\right), 22.8\left(\mathrm{CH}_{2}\right), 27.4\left(\mathrm{CH}_{2}\right)$, $119.3(\mathrm{CH}), 121.0(\mathrm{CH}), 126.4(\mathrm{CH}), 126.8(\mathrm{CH}), 127.2$ (CH), $128.3(\mathrm{CH}), 129.6(\mathrm{CH}), 131.5(\mathrm{C}), 134.7(\mathrm{C}), 136.1$ (C), 136.7 (C), 143.7 (C); HRMS [ESI(+)-TOF] calcd. for $\left[\mathrm{C}_{17} \mathrm{H}_{17} \mathrm{NO}_{2} \mathrm{~S}+\mathrm{H}\right]^{+}$: 300.1053; found: 300.1060 .

(9H-Fluoren-9-yl)methyl (7,8-dihydronaphthalen-2-yl) carbamate (1e)

To a stirred solution of amino ketone $5(0.484 \mathrm{~g}$, $3.00 \mathrm{mmol})$ and pyridine $(0.29 \mathrm{~mL}, 3.60 \mathrm{mmol})$ in anhydrous DCM $(25 \mathrm{~mL})$ at $0{ }^{\circ} \mathrm{C}$ was added solution of Fmoc-Cl (0.854 g, $3.30 \mathrm{mmol})$ in anhydrous DCM and the resulting reaction mixture was allowed to stir at $\mathrm{rt}$. After $1 \mathrm{~h}$ the solution was acidified with $1 \mathrm{~mol} \mathrm{~L}^{-1} \mathrm{HCl}$. The product was extracted with DCM $(3 \times 10 \mathrm{~mL})$ and dried over anhydrous $\mathrm{Mg}_{2} \mathrm{SO}_{4}$. After workup solvent was removed under reduced pressure. The crude product was purified by flash column chromatography (15-25\% EtOAc in hexane) giving Fmoc protected amino ketone 7e. Yield: 1.13 g (98\%); white solid; mp: $160.6-161.5^{\circ} \mathrm{C}$; IR (film) $v_{\max } / \mathrm{cm}^{-1} 3305,3065,2946,2890,1737,1665,1602,1585$, 1537, 1495, 1478, 1450, 1427, 1412, 1350, 1336, 1323, 1287, 1219, 1185, 1164, 1129, 1105; 'H NMR (300 MHz, $\left.\mathrm{CDCl}_{3}\right) \delta 2.06-2.15(\mathrm{~m}, 2 \mathrm{H}), 2.62(\mathrm{t}, 2 \mathrm{H}, J 6.3 \mathrm{~Hz}), 2.91$ (t, 2H, J 6.0 Hz), $4.27(\mathrm{t}, 1 \mathrm{H}, J 6.4 \mathrm{~Hz}, 1 \mathrm{H}), 4.57(\mathrm{~d}, 2 \mathrm{H}$, $J 6.6 \mathrm{~Hz}), 6.95$ (bs, 1H), 7.14 (dd, 1H, J 8.4, 1.5 Hz), 7.32 (td, 1H, J 7.6, 1.2 Hz, 1H), 7.37-7.44 (m, 3H), 7.56-7.62 (m, 2H), $7.78(\mathrm{dt}, 2 \mathrm{H}, J 7.5,0.9 \mathrm{~Hz}), 7.98(\mathrm{~d}, 1 \mathrm{H}, J 8.7 \mathrm{~Hz})$; ${ }^{13} \mathrm{C} \mathrm{NMR}\left(75 \mathrm{MHz}, \mathrm{CDCl}_{3}\right) \delta 23.2\left(\mathrm{CH}_{2}\right), 30.0\left(\mathrm{CH}_{2}\right), 38.9$ $\left(\mathrm{CH}_{2}\right), 47.0(\mathrm{CH}), 67.0\left(\mathrm{CH}_{2}\right), 116.4(\mathrm{CH}), 117.0(\mathrm{CH})$, $120.1(\mathrm{CH}), 124.8(\mathrm{CH}), 127.1(\mathrm{CH}), 127.8(\mathrm{CH}), 128.1$ (C), $128.7(\mathrm{CH}), 141.3(\mathrm{C}), 142.2(\mathrm{C}), 143.5$ (C), 146.3 (C), 152.9 (C), 197.3 (C); HRMS [ESI(+)-TOF] calcd. for $\left[\mathrm{C}_{25} \mathrm{H}_{21} \mathrm{NO}_{3}+\mathrm{H}\right]^{+}: 384.1600$; found: 384.1600 . The reaction 
was performed using ketone $7 \mathrm{e}(1.15 \mathrm{~g}, 3.00 \mathrm{mmol}), \mathrm{MeOH}$ $(50 \mathrm{~mL})$ and $\mathrm{NaBH}_{4}(0.227 \mathrm{~g}, 6.00 \mathrm{mmol})$. After workup, solvent was removed under reduced pressure and crude alcohol (1.12 g, $2.91 \mathrm{mmol}, 97.0 \%)$ was obtained as white solid and used in the next step without purification. The reaction was performed using ( $9 H$-fluoren-9-yl)methyl (5-hydroxy-5,6,7,8-tetrahydronaphthalen-2-yl)carbamate $(1.12 \mathrm{~g}, 2.91 \mathrm{mmol})$, toluene $(50 \mathrm{~mL})$ and $\mathrm{TsOH} . \mathrm{H}_{2} \mathrm{O}$ (cat. few crystals) at $130{ }^{\circ} \mathrm{C}$. The crude product was purified by flash column chromatography (10-30\% EtOAc in hexane) giving alkene 1e. Yield: $0.890 \mathrm{~g}(83 \%)$; white solid; mp: 141-142 ${ }^{\circ} \mathrm{C}$; IR (film) $v_{\max } / \mathrm{cm}^{-1} 3307,3030,2931,2882$, 2826, 1703, 1612, 1585, 1527, 1478, 1465, 1450, 1425, 1326, 1308, 1278, 1219, 1170, 1104; ${ }^{1} \mathrm{H}$ NMR (300 MHz, $\left.\mathrm{CDCl}_{3}\right) \delta 2.25-2.33(\mathrm{~m} \mathrm{2H}), 2.77(\mathrm{t}, 2 \mathrm{H}, J 8.2 \mathrm{~Hz}), 4.27(\mathrm{t}$, $2 \mathrm{H}, J 6.6 \mathrm{~Hz}), 4.53(\mathrm{~d}, 2 \mathrm{H}, J 6.9 \mathrm{~Hz}), 5.95$ (dt, $1 \mathrm{H}, J$ 9.6, $4.4 \mathrm{~Hz}$ ), 6.41 (dt, 1H, J 9.6, 1.6 Hz), 6.57 (bs, 1H), 6.94 (d, $1 \mathrm{H}, J 8.1 \mathrm{~Hz}), 7.08-7.18(\mathrm{~m}, 2 \mathrm{H}), 7.32(\mathrm{td}, 2 \mathrm{H}, J$ 7.4, $1.2 \mathrm{~Hz}), 7.38-7.44$ (m, 2H), 7.61 (d, 2H, J 7.2 Hz), 7.61 (d, $2 \mathrm{H}, J 7.2 \mathrm{~Hz}), 7.78(\mathrm{~d}, 2 \mathrm{H}, J 7.5 \mathrm{~Hz}) ;{ }^{13} \mathrm{C}$ NMR $(75 \mathrm{MHz}$, $\left.\mathrm{CDCl}_{3}\right) \delta 23.0\left(\mathrm{CH}_{2}\right), 27.7\left(\mathrm{CH}_{2}\right), 47.1(\mathrm{CH}), 66.8\left(\mathrm{CH}_{2}\right)$, $116.5(\mathrm{CH}), 118.2(\mathrm{CH}), 120.0(\mathrm{CH}), 124.9(\mathrm{CH}), 126.4$ $(\mathrm{CH}), 127.0(\mathrm{CH}), 127.1(\mathrm{CH}), 127.5(\mathrm{CH}), 127.7(\mathrm{CH})$, 130.0 (C), 136.1 (C), 136.6 (C), 141.3 (C), 143.7 (C), 153.3 (C); HRMS [ESI(+)] calcd. for $\left[\mathrm{C}_{25} \mathrm{H}_{21} \mathrm{NO}_{2}+\mathrm{H}\right]^{+}$: 368.1651; found: 368.1648 .

\section{$\mathrm{N}$-(5,6-Dihydronaphthalen-2-yl)acetamide (1f)}

Compound 1f was prepared according to reported protocol in literature. ${ }^{35}$ Yield: $0.184 \mathrm{~g}(98 \%)$; white solid; mp: 52.1-53.9 ${ }^{\circ} \mathrm{C}$ (lit. 51.2-54.6 ${ }^{\circ} \mathrm{C}$ ).

\section{7,8-Dihydronaphthalen-1-yl acetate $\mathbf{( 9 g})$}

The reaction was performed using ketone $8 \mathrm{~g}(0.892 \mathrm{~g}$, $5.50 \mathrm{mmol}), \mathrm{MeOH}(40 \mathrm{~mL})$ and $\mathrm{NaBH}_{4}(0.208 \mathrm{~g}$, $5.50 \mathrm{mmol}$ ). After workup, solvent was removed under reduced pressure and 1,2,3,4-tetrahydronaphthalene-1,5diol $(0.885 \mathrm{~g}, 5.39 \mathrm{mmol}, 98.0 \%)$ was obtained as white solid and used in the next step without purification. The crude 1,2,3,4-tetrahydronaphthalene-1,5-diol $(0.885 \mathrm{~g}$, $5.39 \mathrm{mmol}$ ), dissolved in anhydrous tetrahydrofuran (THF) $(10 \mathrm{~mL})$ was added with $\mathrm{H}_{3} \mathrm{PO}_{4}(6 \mathrm{~mL})$. The system was maintained at $80^{\circ} \mathrm{C}$ and the reaction was monitored by TLC. The reaction was quenched by addition of saturated solution of $\mathrm{NaHCO}_{3}$ and the mixture was extracted with EtOAc $(3 \times 10 \mathrm{~mL})$. The combined organic extracts were washed with brine and dried over anhydrous $\mathrm{MgSO}_{4}$. The solvent was removed at reduced pressure and the residue was purified by flash chromatography (10-15\% ethyl acetate in hexane) giving alkene 1g. ${ }^{35}$ Yield: $0.614 \mathrm{~g}(78 \%)$; white solid; mp: $66-67^{\circ} \mathrm{C}$ (lit. $53.3-54.6^{\circ} \mathrm{C}^{46}$ and $\left.73.5-74.5^{\circ} \mathrm{C}\right) .{ }^{35}$
To a stirred solution of alkene $1 \mathrm{~g}(0.614 \mathrm{~g}, 4.20 \mathrm{mmol})$ and DMAP $(0.015 \mathrm{~g}, 0.12 \mathrm{mmol})$ in $\mathrm{Et}_{3} \mathrm{~N}(16 \mathrm{~mL})$ was added $\mathrm{Ac}_{2} \mathrm{O}(1.50 \mathrm{~mL}, 15.90 \mathrm{mmol})$ at rt. After $1 \mathrm{~h}$, the reaction was quenched with $\mathrm{MeOH}(5 \mathrm{~mL})$ and $\mathrm{H}_{2} \mathrm{O}(8 \mathrm{~mL})$. The reaction was extracted with EtOAc $(3 \times 10 \mathrm{~mL})$, washed with brine $(2 \times 10 \mathrm{~mL})$ and dried over anhydrous $\mathrm{MgSO}_{4}$. Purification by flash column chromatography (3-5\% EtOAc in hexane) gave acetyl protected alkene $9 g$ g. ${ }^{47}$ Yield: $0.760 \mathrm{~g}$ (96\%); colorless liquid.

\section{7,8-Dihydronaphthalen-1-yl benzoate (9gg)}

The reaction was performed using phenol $1 \mathrm{~g}(0.248 \mathrm{~g}$, $1.70 \mathrm{mmol}), \mathrm{BzCl}(0.22 \mathrm{~mL}, 0.267 \mathrm{~g}, 1.90 \mathrm{mmol})$ and $\mathrm{Et}_{3} \mathrm{~N}(0.51 \mathrm{~mL}, 0.374 \mathrm{~g}, 3.70 \mathrm{mmol})$ in DCM $(10 \mathrm{~mL})$. Purification by flash column chromatography (3-4\% EtOAc in hexane) gave benzoyl protected alkene 9gg. Yield: 0.398 g (94\%); white solid; mp: 58.1-58.7 ${ }^{\circ} \mathrm{C}$; IR (film) $v_{\max } / \mathrm{cm}^{-1} 3035,2935,2887,2834,2127,1735,1651,1601$, $1583,1569,1491,1451,1395,1342,1314,1296,1266$, $1247,1229,1212 ;{ }^{1} \mathrm{H} \mathrm{NMR}\left(300 \mathrm{MHz}, \mathrm{CDCl}_{3}\right) \delta 2.24-2.32$ (m, 2H), $2.71(\mathrm{t}, 2 \mathrm{H}, J 8.4 \mathrm{~Hz}), 6.04(\mathrm{dt}, 1 \mathrm{H}, J 9.6,4.5 \mathrm{~Hz})$, 6.49 (dt, 1H, J 9.6, $1.8 \mathrm{~Hz}), 6.96$ (d, 1H, J 7.5 Hz), 7.00 (dd, $1 \mathrm{H}, J$ 8.1, $1.2 \mathrm{~Hz}), 7.17-7.22(\mathrm{~m}, 1 \mathrm{H}), 7.48-7.54(\mathrm{~m}$, $2 \mathrm{H}), 7.61-7.66(\mathrm{~m}, 1 \mathrm{H}), 8.21-8.25(\mathrm{~m}, 2 \mathrm{H}) ;{ }^{13} \mathrm{C} \mathrm{NMR}$ $\left(75 \mathrm{MHz}, \mathrm{CDCl}_{3}\right) \delta 20.6\left(\mathrm{CH}_{2}\right), 22.3\left(\mathrm{CH}_{2}\right), 120.8(\mathrm{CH})$, $123.8(\mathrm{CH}), 126.8(\mathrm{CH}), 127.2(\mathrm{C}), 127.3(\mathrm{CH}), 128.6$ $(\mathrm{CH}), 129.0(\mathrm{CH}), 129.4(\mathrm{C}), 130.1(\mathrm{CH}), 133.5(\mathrm{CH})$, 135.7 (C), 148.0 (C), 164.8 (C); LRMS m/z (rel. int.): 250 $\left(\mathrm{M}^{\bullet}, 9\right), 144$ (3), 128(11), 115 (10), 105 (100), 91 (2), 77 (36), 63 (3), 51 (10), 39 (2); HRMS [ESI(+)-TOF] calcd. for $\left[\mathrm{C}_{17} \mathrm{H}_{14} \mathrm{O}_{2}+\mathrm{Na}\right]^{+}:$273.0891; found: 273.0890 .

\section{7,8-Dihydronaphthalen-2-yl acetate (9h)}

The reaction was performed using ketone $\mathbf{8 h}(2.000 \mathrm{~g}$, $11.35 \mathrm{mmol}), \mathrm{MeOH}(80 \mathrm{~mL})$ and $\mathrm{NaBH}_{4}(0.4290 \mathrm{~g}$, $11.35 \mathrm{mmol}$ ). After workup, solvent was removed under reduced pressure and crude alcohol $(1.986 \mathrm{~g}, 11.14 \mathrm{mmol}$, 98.0\%) was obtained as white solid and used in the next step without purification. The reaction was performed following using 6-methoxy-1,2,3,4-tetrahydronaphthalen-1ol $(1.986 \mathrm{~g}, 11.14 \mathrm{mmol})$, toluene $(60 \mathrm{~mL})$ and $\mathrm{TsOH} . \mathrm{H}_{2} \mathrm{O}$ (cat. few crystals) at $130{ }^{\circ} \mathrm{C}$. The crude product was purified by flash column chromatography (20-40\% EtOAc in hexane) giving alkene $1 \mathbf{h} .{ }^{36}$ Yield: $1.702 \mathrm{~g}(95 \%)$; colorless oil. Under inert atmosphere $\mathrm{N}_{2}, \mathrm{NaH}(6.24 \mathrm{~g}, 260 \mathrm{mmol}, 60 \%$ in mineral oil) was washed with anhydrous hexanes $(3 \times 20 \mathrm{~mL})$. After a few minutes, anhydrous DMF ( $75 \mathrm{~mL}$ ) was added. To this mixture was slowly added a solution of EtSH $(13.0 \mathrm{~mL}$, $180 \mathrm{mmol}, 30$ equiv based on olefin $\mathbf{1 h}$ ) in anhydrous DMF $(14 \mathrm{~mL})$ at $0{ }^{\circ} \mathrm{C}$ and the resulting yellow solution was stirred for $20 \mathrm{~min}$ at rt. A solution of alkene $1 \mathrm{~h}(0.961 \mathrm{~g}, 6.0 \mathrm{mmol})$ 
in DMF $(15 \mathrm{~mL})$ was then added dropwise and the resulting mixture was stirred for $5 \mathrm{~h}$ at $140{ }^{\circ} \mathrm{C}$. When the reaction was becoming slightly brown, the mixture was cooled to the $\mathrm{rt}$ and a saturated solution of $\mathrm{NH}_{4} \mathrm{Cl}$ was added. The mixture was extracted with $\mathrm{Et}_{2} \mathrm{O}$. The organic phase was washed with water, brine and dried over anhydrous $\mathrm{MgSO}_{4}$. The solvent was removed under reduced pressure. The crude product was purified by flash column chromatography (20-40\% EtOAc in hexane) giving 7,8-dihydronaphthalen-2-ol. ${ }^{39}$ Yield: $0.851 \mathrm{~g}$ (97\%); viscous colorless liquid. The reaction was performed using 7,8-dihydronaphthalen-2-ol (0.365 g, $2.50 \mathrm{mmol})$, DMAP $(0.09 \mathrm{~g}, 0.07 \mathrm{mmol})$ and $\mathrm{Ac}_{2} \mathrm{O}(0.9 \mathrm{~mL}, 9.5 \mathrm{mmol})$ in $\mathrm{Et}_{3} \mathrm{~N}(10 \mathrm{~mL})$. Purification by flash column chromatography (3-5\% EtOAc in hexane) gave acetyl protected alkene $\mathbf{9 h}{ }^{48}$ Yield: $0.398 \mathrm{~g}(85 \%)$; colorless liquid.

\section{5,6-Dihydronaphthalen-2-yl acetate (9b)}

The reaction was performed using ketone $\mathbf{8 b}(0.705 \mathrm{~g}$, $4.00 \mathrm{mmol}), \mathrm{MeOH}(40 \mathrm{~mL})$ and $\mathrm{NaBH}_{4}(0.152 \mathrm{~g}$, $4.00 \mathrm{mmol})$. After workup, solvent was removed under reduced pressure and crude alcohol $(0.691 \mathrm{~g}, 3.88 \mathrm{mmol}$, $97 \%$ ) was obtained as white solid and used in the next step without purification. The reaction was performed using 7-methoxy-1,2,3,4-tetrahydronaphthalen-1-ol (0.691 g, $3.88 \mathrm{mmol})$, toluene $(40 \mathrm{~mL})$ and $\mathrm{TsOH} . \mathrm{H}_{2} \mathrm{O}$ (cat. few crystals) at $130{ }^{\circ} \mathrm{C}$. After workup solvent was removed under reduced pressure. The crude product was purified by flash column chromatography (1-2\% EtOAc in hexane) giving alkene 1b. ${ }^{13,37}$ Yield: $0.488 \mathrm{~g}$ (78\%); colorless liquid. The reaction was performed using alkene 1 b (0.481 g, $3.00 \mathrm{mmol})$, NaH (3.12 g, $130 \mathrm{mmol}, 60 \%$ in mineral oil), EtSH (6.5 mL, 90 mmol, 30 equiv. based on olefin 1b) in DMF (35 mL) at $140{ }^{\circ} \mathrm{C}$. The crude product was purified by flash column chromatography (10-15\% ethyl acetate in hexane) giving 5,6-dihydronaphthalen2-ol. Yield: $0.351 \mathrm{~g}(80 \%)$; white solid; $\mathrm{mp}$ : $98-99{ }^{\circ} \mathrm{C}$; IR (film) $v_{\max } / \mathrm{cm}^{-1} 3247,3027,2936,2880,2851,2818$, 1629, 1614, 1574, 1492, 1477, 1465, 1435, 1426, 1395, 1349, 1327, 1282, 1265, 1215; ${ }^{1} \mathrm{H} \mathrm{NMR}\left(300 \mathrm{MHz}, \mathrm{CDCl}_{3}\right)$ $\delta 2.25-2.32(\mathrm{~m}, 2 \mathrm{H}), 2.71(\mathrm{t}, 2 \mathrm{H}, J 8.1 \mathrm{~Hz}), 4.59(\mathrm{~s}, 1 \mathrm{H})$, $6.04(\mathrm{dt}, 1 \mathrm{H}, J 9.6,4.4 \mathrm{~Hz}), 6.38(\mathrm{dt}, 1 \mathrm{H}, J 9.6,1.8 \mathrm{~Hz})$, $6.52(\mathrm{~d}, 1 \mathrm{H}, J 2.7 \mathrm{~Hz}), 6.59$ (dd, 1H, J 7.8, 2.7 Hz), 6.96 (d, $1 \mathrm{H}, J$ 8.1, Hz); ${ }^{13} \mathrm{C} \mathrm{NMR}\left(75 \mathrm{MHz}, \mathrm{CDCl}_{3}\right) \delta 23.5\left(\mathrm{CH}_{2}\right)$, $26.6\left(\mathrm{CH}_{2}\right), 112.9(\mathrm{CH}), 113.1(\mathrm{CH}), 127.5(\mathrm{CH}), 127.7(\mathrm{C})$, $128.3(\mathrm{CH}), 129.5(\mathrm{CH}), 135.3(\mathrm{C}), 154.0(\mathrm{C})$; LRMS m/z (rel. int.): $146\left(\mathrm{M}^{+\bullet}, 100\right), 145$ (71), 131 (45), 127 (43), 117 (36), 115 (55), 103 (5), 91 (12), 77 (7), 63 (14), 51 (13), 39 (10); HRMS [ESI(+)-TOF] calcd. for $\left[\mathrm{C}_{10} \mathrm{H}_{10} \mathrm{O}+\mathrm{K}\right]^{+}$: 185.0369; found: 185.0361. The reaction was performed using 5,6-dihydronaphthalen-2-ol $(0.351 \mathrm{~g}, 2.40 \mathrm{mmol})$, DMAP $(0.08 \mathrm{~g}, 0.065 \mathrm{mmol})$ and $\mathrm{Ac}_{2} \mathrm{O}(0.9 \mathrm{~mL}, 9.0 \mathrm{mmol})$ in $\mathrm{Et}_{3} \mathrm{~N}(10 \mathrm{~mL})$. Purification by flash column chromatography (3-5\% EtOAc in hexane) gave acetyl protected alkene 9b. Yield: $0.359 \mathrm{~g}(85 \%)$; colorless liquid; IR (film) $v_{\max } / \mathrm{cm}^{-1} 3034,2935,2885,2831,1761,1609$, 1575, 1491, 1432, 1369, 1328, 1271, 1210; ${ }^{1} \mathrm{H}$ NMR $\left(300 \mathrm{MHz}, \mathrm{CDCl}_{3}\right) \delta 2.27-2.36(\mathrm{~m}, 2 \mathrm{H}), 2.28(\mathrm{~s}, 3 \mathrm{H}), 2.77$ $(\mathrm{t}, 2 \mathrm{H}, J 8.0 \mathrm{~Hz}), 6.06(\mathrm{~m}, 1 \mathrm{H}), 6.41(\mathrm{dt}, 1 \mathrm{H}, J 9.6,1.8 \mathrm{~Hz})$, $6.74(\mathrm{~d}, 1 \mathrm{H}, J 2.4 \mathrm{~Hz}), 6.81(\mathrm{dd}, 1 \mathrm{H}, J 8.1,2.4 \mathrm{~Hz}), 7.08$ (d, $1 \mathrm{H}, J 8.1 \mathrm{~Hz}) ;{ }^{13} \mathrm{C} \mathrm{NMR}\left(75 \mathrm{MHz}, \mathrm{CDCl}_{3}\right) \delta 21.1\left(\mathrm{CH}_{3}\right)$, $23.2\left(\mathrm{CH}_{2}\right), 26.8\left(\mathrm{CH}_{2}\right), 118.8(\mathrm{CH}), 119.4(\mathrm{CH}), 127.3$ (CH), $128.2(\mathrm{CH}), 129.6(\mathrm{CH}), 132.9(\mathrm{C}), 135.3(\mathrm{C}), 149.2$ (C), 169.7 (C); LRMS m/z (rel. int.): $188\left(\mathrm{M}^{+\bullet}, 20\right), 146$ (100), 145 (52), 131 (30), 127 (19), 117 (24), 115 (42), 91 (15), 77 (5), 63 (9), 43 (30), 39 (10); HRMS [ESI(+)] calcd. for $\left[\mathrm{C}_{12} \mathrm{H}_{12} \mathrm{O}_{2}+\mathrm{Na}\right]^{+}:$211.0735; found: 211.0724 .

Ring contraction reactions mediated by HTIB: general procedure for $\mathbf{2 b}, \mathbf{2 g}, \mathbf{2} \mathbf{g g}$ and $\mathbf{2 h}$

1-(Bis(2,2,2-trifluoroethoxy)methyl)-2,3-dihydro- $1 \mathrm{H}$-inden4-yl acetate $(\mathbf{2 g})$

To a solution of alkene $9 \mathrm{~g}(0.094 \mathrm{~g}, 0.50 \mathrm{mmol})$ in TFE $(10 \mathrm{~mL})$ was added HTIB $(0.235 \mathrm{~g}, 0.60 \mathrm{mmol})$ at $0{ }^{\circ} \mathrm{C}$. The reaction mixture was stirred at $0{ }^{\circ} \mathrm{C}$ for $5 \mathrm{~min}$. The reaction was quenched with a saturated solution of $\mathrm{NaHCO}_{3}$. The reaction mixture was extracted with EtOAc $(3 \times 15 \mathrm{~mL})$, washed with brine $(2 \times 10 \mathrm{~mL})$ and dried over anhydrous $\mathrm{MgSO}_{4}$. The solvent was evaporated under reduced pressure. The crude product was purified by flash column chromatography (4-5\% EtOAc in hexanes) giving acetal 2g. Yield: $0.128 \mathrm{~g}(66 \%)$; white solid; mp: 61.6-63.4 ${ }^{\circ} \mathrm{C}$; IR (film) $v_{\max } / \mathrm{cm}^{-1} 2947,1764,1615,1587,1468,1433$, 1372, 1281, 1213; ${ }^{1} \mathrm{H}$ NMR (300 MHz, $\left.\mathrm{CDCl}_{3}\right) \delta 1.98-2.10$ $(\mathrm{m}, 1 \mathrm{H}), 2.22-2.32(\mathrm{~m}, 1 \mathrm{H}), 2.30(\mathrm{~s}, 3 \mathrm{H}), 2.70-2.91(\mathrm{~m}$, 2H), 3.47-3.55 (m, 1H), 3.84-4.07 (m, 4H), $4.71(\mathrm{~d}, 1 \mathrm{H}$, $J 8.1 \mathrm{~Hz}), 6.94(\mathrm{dt}, 1 \mathrm{H}, J 7.8 \mathrm{~Hz}), 7.21(\mathrm{t}, 1 \mathrm{H}, J 7.8 \mathrm{~Hz}), 7.28$ $(\mathrm{d}, 1 \mathrm{H}, J 7.5 \mathrm{~Hz}) ;{ }^{13} \mathrm{C} \mathrm{NMR}\left(75 \mathrm{MHz}, \mathrm{CDCl}_{3}\right) \delta 20.8\left(\mathrm{CH}_{3}\right)$, $26.8\left(\mathrm{CH}_{2}\right), 28.1\left(\mathrm{CH}_{2}\right), 47.5(\mathrm{CH}), 61.8(\mathrm{q}, J 34.8 \mathrm{~Hz})$ $\left(\mathrm{CH}_{2}\right), 63.3(\mathrm{q}, J 34.8 \mathrm{~Hz})\left(\mathrm{CH}_{2}\right), 105.0(\mathrm{CH}), 120.6(\mathrm{CH})$, $123.2(\mathrm{CH}), 123.6(\mathrm{q}, J 276 \mathrm{~Hz})\left(\mathrm{CF}_{3}\right), 123.7(\mathrm{q}, J 276 \mathrm{~Hz})$ $\left(\mathrm{CF}_{3}\right), 128.0(\mathrm{CH}), 136.6(\mathrm{C}), 143.3(\mathrm{C}), 147.1(\mathrm{C}), 168.9$ (C); HRMS [ESI(+)-TOF] calcd. for $\left[\mathrm{C}_{16} \mathrm{H}_{16} \mathrm{~F}_{6} \mathrm{O}_{4}+\mathrm{Na}\right]^{+}$: 409.0850; found: 409.0851 .

1-(Bis(2,2,2-trifluoroethoxy)methyl)-2,3-dihydro-1 $H$-inden4-yl benzoate (2gg)

Yield: $0.159 \mathrm{~g}(71 \%)$; white solid; mp: $79-79.8^{\circ} \mathrm{C}$; IR (film) $v_{\max } / \mathrm{cm}^{-1} 3067,2948,1738,1602,1585,1469,1453$, 1423, 1383, 1279, 1230, 1168, 1134; ${ }^{1} \mathrm{H}$ NMR (300 MHz, $\left.\mathrm{CDCl}_{3}\right) \delta 1.99-2.11(\mathrm{~m}, 1 \mathrm{H}), 2.20-2.32(\mathrm{~m}, 1 \mathrm{H}), 2.76-2.97$ (m, 2H), 3.54 (q, 1H, J 8.1 Hz), 3.85-4.09 (m, 4H), 4.74 
(d, 1H, J 8.1 Hz), $7.10(\mathrm{dt}, 1 \mathrm{H}, J 7.8,1.2 \mathrm{~Hz}), 7.26(\mathrm{t}$, $1 \mathrm{H}, J 7.8 \mathrm{~Hz}), 7.33$ (d, 1H, J 7.8 Hz), 7.48-7.54 (m, 2H), $7.64(\mathrm{tt}, 1 \mathrm{H}, J 7.5,1.5 \mathrm{~Hz}), 8.18-8.22(\mathrm{~m}, 2 \mathrm{H}) ;{ }^{13} \mathrm{C} \mathrm{NMR}$ $\left(75 \mathrm{MHz}, \mathrm{CDCl}_{3}\right) \delta 26.8\left(\mathrm{CH}_{2}\right), 28.2\left(\mathrm{CH}_{2}\right), 47.6(\mathrm{CH})$, $61.9(\mathrm{q}, J 34.8 \mathrm{~Hz})\left(\mathrm{CH}_{2}\right), 63.4(\mathrm{q}, J 34.8 \mathrm{~Hz})\left(\mathrm{CH}_{2}\right)$, $105.1(\mathrm{CH}), 120.7(\mathrm{CH}), 123.2(\mathrm{CH}), 123.69(\mathrm{q}, J 276 \mathrm{~Hz})$ $\left(\mathrm{CF}_{3}\right), 123.74(\mathrm{q}, J 276 \mathrm{~Hz})\left(\mathrm{CF}_{3}\right), 128.1(\mathrm{CH}), 128.6(\mathrm{CH})$, $129.4(\mathrm{C}), 130.2(\mathrm{CH}), 133.6(\mathrm{CH}), 136.8(\mathrm{C}), 143.4(\mathrm{C})$, 147.4 (C), 164.5 (C); HRMS [ESI(+)-TOF]: calcd. for $\left[\mathrm{C}_{21} \mathrm{H}_{18} \mathrm{~F}_{6} \mathrm{O}_{4}+\mathrm{Na}\right]^{+}$: 471.1007; found: 471.1010.

1-(Bis(2,2,2-trifluoroethoxy)methyl)-2,3-dihydro- $1 H$-inden5 -yl acetate $(\mathbf{2 h})$

Yield: $0.117 \mathrm{~g}(60 \%)$; viscous colorless liquid; IR (film) $v_{\max } / \mathrm{cm}^{-1} 2953,1761,1610,1592,1485,1460,1431,1372$, $1282,1216,1163,1134,1103 ;{ }^{1} \mathrm{H} N \mathrm{MR}\left(300 \mathrm{MHz}, \mathrm{CDCl}_{3}\right)$ $\delta$ 1.97-2.09 (m, 1H), 2.21-2.33 (m, 1H), $2.28(\mathrm{~s}, 3 \mathrm{H}), 2.82-$ $3.01(\mathrm{~m}, 2 \mathrm{H}), 3.43(\mathrm{q}, 1 \mathrm{H}, J 7.8 \mathrm{~Hz}), 3.86-4.08(\mathrm{~m}, 4 \mathrm{H})$, 4.68 (d, 1H, J 8.4 Hz), 6.87 (dd, 1H, J 8.4, $2.2 \mathrm{~Hz}), 6.94$ (s, $1 \mathrm{H}), 7.38(\mathrm{~d}, 1 \mathrm{H}, J 8.1 \mathrm{~Hz}) ;{ }^{13} \mathrm{C} \mathrm{NMR}\left(75 \mathrm{MHz}, \mathrm{CDCl}_{3}\right)$ $\delta 21.0\left(\mathrm{CH}_{3}\right), 27.4\left(\mathrm{CH}_{2}\right), 31.1\left(\mathrm{CH}_{2}\right), 46.4(\mathrm{CH}), 61.6(\mathrm{q}$, $J 34.8 \mathrm{~Hz})\left(\mathrm{CH}_{2}\right), 63.3(\mathrm{q}, J 34.8 \mathrm{~Hz})\left(\mathrm{CH}_{2}\right), 105.1(\mathrm{CH})$, $117.8(\mathrm{CH}), 119.7(\mathrm{CH}), 123.6(\mathrm{q}, J 276 \mathrm{~Hz})\left(\mathrm{CF}_{3}\right), 123.7$ (q, J $276 \mathrm{~Hz})\left(\mathrm{CF}_{3}\right), 126.1(\mathrm{CH}), 138.3(\mathrm{C}), 146.1(\mathrm{C}), 150.3$ (C), 169.7 (C); LRMS m/z (rel. int.): $386\left(\mathrm{M}^{+\bullet}, 4\right), 344$ (7), 245 (7), 211 (14), 175 (8), 145 (6), 133 (100), 115 (7), 105 (11), 83 (11), 77 (6), 43 (19); HRMS [ESI(+)-TOF]: calcd. for $\left[\mathrm{C}_{16} \mathrm{H}_{16} \mathrm{~F}_{6} \mathrm{O}_{4}+\mathrm{Na}\right]^{+}: 409.0850$; found: 409.0851 .

3-(Bis(2,2,2-trifluoroethoxy)methyl)-2,3-dihydro- $1 H$-inden5 -yl acetate $(\mathbf{2 b})$

Yield: $0.126 \mathrm{~g}(65 \%)$; white solid; mp: $63.6-65.2{ }^{\circ} \mathrm{C}$; IR (film) $v_{\max } / \mathrm{cm}^{-1} 2919,2850,1760,1610,1591,1540,1484$, $1459,1429,1372,1281,1214 ;{ }^{1} \mathrm{H}$ NMR $\left(300 \mathrm{MHz}, \mathrm{CDCl}_{3}\right)$ $\delta$ 1.98-2.09 (m, 1H), 2.21-2.33 (m, 1H), 2.28 (s, 3H), 2.79$2.99(\mathrm{~m}, 2 \mathrm{H}), 3.46(\mathrm{q}, 1 \mathrm{H}, J 7.8 \mathrm{~Hz}), 3.85-4.06(\mathrm{~m}, 2 \mathrm{H})$, 4.69 (d, 1H, $J 8.4 \mathrm{~Hz}$ ), 6.91 (ddd, $1 \mathrm{H}, J 8.1,2.1,0.3 \mathrm{~Hz}$ ), $7.10(\mathrm{~d}, 1 \mathrm{H}, J 1.8 \mathrm{~Hz}), 7.20(\mathrm{~d}, 1 \mathrm{H}, J 8.1 \mathrm{~Hz}) ;{ }^{13} \mathrm{C} \mathrm{NMR}$ $\left(75 \mathrm{MHz}, \mathrm{CDCl}_{3}\right) \delta 21.1\left(\mathrm{CH}_{3}\right), 27.5\left(\mathrm{CH}_{2}\right), 30.6\left(\mathrm{CH}_{2}\right), 61.7$ (q, J $34.8 \mathrm{~Hz})\left(\mathrm{CH}_{2}\right), 63.5(\mathrm{q}, J 34.8 \mathrm{~Hz})\left(\mathrm{CH}_{2}\right), 105.1(\mathrm{CH})$, $118.9(\mathrm{CH}), 120.9(\mathrm{CH}), 123.6(\mathrm{q}, J 276 \mathrm{~Hz})\left(\mathrm{CF}_{3}\right), 123.7$ (q, $J 276 \mathrm{~Hz})\left(\mathrm{CF}_{3}\right), 125.1(\mathrm{CH}), 142.0(\mathrm{C}), 142.2(\mathrm{C}), 149.5$ (C), 169.8 (C); LRMS m/z (rel. int.): $386\left(\mathrm{M}^{+\bullet}, 2\right), 344$ (25), 287 (7), 244 (8), 211 (100), 145 (17), 133 (92), 115 (15), 105 (17), 83 (29), 77 (12), 43 (43); HRMS [ESI(+)-TOF] calcd. for $\left[\mathrm{C}_{16} \mathrm{H}_{16} \mathrm{~F}_{6} \mathrm{O}_{4}+\mathrm{Na}\right]^{+}$: 409.0850; found: 409.0851 .

$\mathrm{N}$-(1-(Dimethoxymethyl)-2,3-dihydro-1H-inden-5-yl) benzamide (3c)

To a solution of alkene $11(0.125 \mathrm{~g}, 0.500 \mathrm{mmol})$ in $\mathrm{MeOH} / \mathrm{DCM}$ (8:1) (10 mL) was added HTIB (0.235 g,
$0.60 \mathrm{mmol}$ ) at $0{ }^{\circ} \mathrm{C}$ for $10 \mathrm{~min}$. The reaction was quenched with a saturated solution of $\mathrm{NaHCO}_{3}$. The reaction mixture was extracted with EtOAc $(3 \times 15 \mathrm{~mL})$, washed with brine $(2 \times 10 \mathrm{~mL})$ and dried over anhydrous $\mathrm{MgSO}_{4}$. The solvent was evaporated under reduced pressure. The crude product was purified by flash column chromatography (40-50\% EtOAc in hexane), giving acetal 3c. Yield: $0.12 \mathrm{~g}(77 \%)$; yellowish white solid; mp: $96-97.5^{\circ} \mathrm{C}$; IR (film) $v_{\max } / \mathrm{cm}^{-1}$ 3307, 3060, 2937, 2830, 1737, 1650, 1601, 1580, 1532, 1493, $1447,1424,1374,1328,1283,1248,1210,1187,1154,1116$; ${ }^{1} \mathrm{H} \mathrm{NMR}\left(300 \mathrm{MHz}, \mathrm{CDCl}_{3}\right) \delta 1.92-2.04(\mathrm{~m}, 1 \mathrm{H}), 2.15-2.27$ (m, 1H), 2.78-2.99 (m, 2H), 3.37-3.47 (m, 1H), $3.38(\mathrm{~s}, 3 \mathrm{H})$, $3.42(\mathrm{~s}, 3 \mathrm{H}), 4.29$ (d, 1H, J 7.5 Hz), 7.29 (dd, 1H, J 8.1, $2.1 \mathrm{~Hz}$ ), 7.38 (d, 1H, J 8.4 Hz), 7.42-7.55 (m, 3H), 7.61 (s, 1H), 7.83-7.86 (m, 2H), $7.93(\mathrm{bs}, 1 \mathrm{H}) ;{ }^{13} \mathrm{C} \mathrm{NMR}(75 \mathrm{MHz}$, $\left.\mathrm{CDCl}_{3}\right) \delta 27.5\left(\mathrm{CH}_{2}\right), 31.4\left(\mathrm{CH}_{2}\right), 46.9(\mathrm{CH}), 52.8\left(\mathrm{CH}_{3}\right), 54.2$ $\left(\mathrm{CH}_{3}\right), 107.1(\mathrm{CH}), 116.6(\mathrm{CH}), 118.5(\mathrm{CH}), 125.6(\mathrm{CH})$, $126.9(\mathrm{CH}), 128.5(\mathrm{CH}), 131.5(\mathrm{CH}), 134.9(\mathrm{C}), 136.8(\mathrm{C})$, 139.1 (C), 145.7 (C), 165.8 (C); HRMS [ESI(+)]: calcd. for $\left[\mathrm{C}_{19} \mathrm{H}_{21} \mathrm{NO}_{3}+\mathrm{H}\right]^{+}$: 312.1600; found: 312.1600 .

(9H-Fluoren-9-yl)methyl (1-(dimethoxymethyl)-2,3-dihydro$1 H$-inden-5-yl)carbamate (3e)

The reaction was performed using alkene $1 \mathrm{e}(0.183 \mathrm{~g}$, $0.50 \mathrm{mmol})$, HTIB (0.235 g, $0.60 \mathrm{mmol})$ and $\mathrm{MeOH} / \mathrm{DCM}$ (4:1) $(10 \mathrm{~mL})$ at $0{ }^{\circ} \mathrm{C}$ for $10 \mathrm{~min} .{ }^{13}$ The crude product was purified by flash column chromatography (15-25\% EtOAc in hexane) giving acetal 3e. Yield: $0.138 \mathrm{~g}(64 \%)$; white solid; mp: $115-117^{\circ} \mathrm{C}$; IR (film) $v_{\max } / \mathrm{cm}^{-1} 3307,3066,2942,2849$, 2830, 1730, 1708, 1598, 1538, 1493, 1478, 1450, 1431, 1375, 1326, 1297, 1220; ${ }^{1} \mathrm{H}$ NMR (300 MHz, $\left.\mathrm{CDCl}_{3}\right) \delta 1.89-2.01$ $(\mathrm{m}, 1 \mathrm{H}), 2.12-2.24(\mathrm{~m}, 1 \mathrm{H}), 2.73-2.94(\mathrm{~m}, 2 \mathrm{H}), 3.34-3.43(\mathrm{~m}$, $1 \mathrm{H}), 3.35(\mathrm{~s}, 3 \mathrm{H}), 3.40(\mathrm{~s}, 3 \mathrm{H}), 4.22-4.26(\mathrm{~m}, 1 \mathrm{H}), 4.26(\mathrm{~d}$, $1 \mathrm{H}, J 7.5 \mathrm{~Hz}), 4.51$ (d, 2H, J 6.6 Hz), 6.71 (bs, 1H), 7.04 (d, $1 \mathrm{H}, J 7.2 \mathrm{~Hz}), 7.27-7.32(\mathrm{~m}, 4 \mathrm{H}), 7.36-7.41(\mathrm{~m}, 2 \mathrm{H}), 7.59$ (d, 2H, J 7.5 Hz), 7.76 (d, 2H, J 7.5 Hz); ${ }^{13} \mathrm{C} \mathrm{NMR}(75 \mathrm{MHz}$, $\left.\mathrm{CDCl}_{3}\right) \delta 27.5\left(\mathrm{CH}_{2}\right), 31.4\left(\mathrm{CH}_{2}\right), 46.8(\mathrm{CH}), 47.0(\mathrm{CH}), 52.8$ $\left(\mathrm{CH}_{3}\right), 54.1\left(\mathrm{CH}_{3}\right), 66.6\left(\mathrm{CH}_{2}\right), 107.1(\mathrm{CH}), 115.0(\mathrm{CH})$, $117.0(\mathrm{CH}), 119.9(\mathrm{CH}), 124.8(\mathrm{CH}), 125.6(\mathrm{CH}), 127.0$ $(\mathrm{CH}), 127.6(\mathrm{CH}), 136.6(\mathrm{C}), 138.0(\mathrm{C}), 141.2(\mathrm{C}), 143.7$ (C), 145.7 (C), 153.5 (C); HRMS [ESI(+)-TOF]: calcd. for $\left[\mathrm{C}_{27} \mathrm{H}_{27} \mathrm{NO}_{4}+\mathrm{Na}\right]^{+}$: 452.1832; found: 452.1836 .

$N$-((5R,6R)-5,6-Dimethoxy-5,6,7,8-tetrahydronaphthalen-2yl)-4-methylbenzenesulfonamide (trans-4d) and $N-((5 R, 6 S)$ 5,6-dimethoxy-5,6,7,8-tetrahydronaphthalen-2-yl)-4methylbenzenesulfonamide (cis-4d)

The reaction was performed using alkene $1 \mathbf{d}(0.150 \mathrm{~g}$, $0.50 \mathrm{mmol})$, HTIB $(0.235 \mathrm{~g}, 0.60 \mathrm{mmol})$ and $\mathrm{MeOH}$ $(10 \mathrm{~mL})$ at $0{ }^{\circ} \mathrm{C} .{ }^{13}$ The crude product was purified by flash column chromatography (20-50\% EtOAc in hexane) giving 
trans $-\mathbf{4 d}$, cis-4d and starting material $\mathbf{1 d}(0.021 \mathrm{~g}, 0.070$ mmol, 14\%) was recovered.

$N-((5 R, 6 R)-5,6$-Dimethoxy-5,6,7,8-tetrahydronaphthalen2-yl)-4-methylbenzenesulfonamide (trans-4d)

Yield: $0.019 \mathrm{~g}(10 \%)$; viscous colorless oil; IR (film) $v_{\max } / \mathrm{cm}^{-1} 3257,2932,2825,1919,1614,1599,1502$, 1463, 1399, 1341, 1320, 1292, 1270, 1185, 1162; ' ${ }^{H}$ NMR $\left(300 \mathrm{MHz}, \mathrm{CDCl}_{3}\right) \delta 1.82-1.92(\mathrm{~m}, 1 \mathrm{H}), 1.99-2.09(\mathrm{~m}, 1 \mathrm{H})$, $2.37(\mathrm{~s}, 3 \mathrm{H}), 2.57-2.80(\mathrm{~m}, 2 \mathrm{H}), 3.42(\mathrm{~s}, 3 \mathrm{H}), 3.48(\mathrm{~s}, 3 \mathrm{H})$, 3.65-3.70 (m, 1H), $4.14(\mathrm{~d}, 1 \mathrm{H}, J 4.8 \mathrm{~Hz}), 6.82-6.88(\mathrm{~m}$, $3 \mathrm{H}), 7.17-7.23(\mathrm{~m}, 3 \mathrm{H}), 7.67$ (d, 2H, J 8.7 Hz); ${ }^{13} \mathrm{C} \mathrm{NMR}$ $\left(75 \mathrm{MHz}, \mathrm{CDCl}_{3}\right) \delta 21.5\left(\mathrm{CH}_{3}\right), 22.9\left(\mathrm{CH}_{2}\right), 25.1\left(\mathrm{CH}_{2}\right)$, $56.5\left(\mathrm{CH}_{3}\right), 57.6\left(\mathrm{CH}_{3}\right), 77.5(\mathrm{CH}), 79.1(\mathrm{CH}), 118.5(\mathrm{CH})$, $120.5(\mathrm{CH}), 127.2(\mathrm{CH}), 129.6(\mathrm{CH}), 130.9(\mathrm{CH}), 131.4$ (C), 135.9 (C), 136.2 (C), 138.2 (C), 143.7 (C); HRMS [ESI(+)-TOF]: calcd. for $\left[\mathrm{C}_{19} \mathrm{H}_{23} \mathrm{NO}_{4} \mathrm{~S}+\mathrm{K}\right]^{+}$: 400.0985; found: 400.0988 .

$N-((5 R, 6 S)-5,6-$ Dimethoxy-5,6,7,8-tetrahydronaphthalen-2yl)-4-methylbenzenesulfonamide (cis-4d)

Yield: $0.068 \mathrm{~g}$ (37\%); viscous colorless oil; IR (film) $v_{\max } / \mathrm{cm}^{-1} 3249,3065,2932,2827,1919,1674,1647$, 1612, 1500, 1463, 1399, 1340, 1320, 1292, 1230; ${ }^{1} \mathrm{H}$ NMR $\left(300 \mathrm{MHz}, \mathrm{CDCl}_{3}\right) \delta 1.82-1.90(\mathrm{~m}, 1 \mathrm{H}), 2.08-2.18(\mathrm{~m}, 1 \mathrm{H})$, $2.36(\mathrm{~s}, 1 \mathrm{H}), 2.60-2.71(\mathrm{~m}, 1 \mathrm{H}), 2.84-2.91(\mathrm{~m}, 1 \mathrm{H}), 3.42(\mathrm{~s}$, $3 \mathrm{H}), 3.44$ (s, 3H), 3.59 (dt, $1 \mathrm{H}, J 9.9,3.0 \mathrm{~Hz}), 4.26(\mathrm{~d}, 1 \mathrm{H}$, $J 2.7 \mathrm{~Hz}), 6.86(\mathrm{~d}, 1 \mathrm{H}, J 2.1 \mathrm{~Hz}), 6.90(\mathrm{dd}, 1 \mathrm{H}, J 8.1,2.4 \mathrm{~Hz})$, $7.15(\mathrm{~d}, 1 \mathrm{H}, J 8.4 \mathrm{~Hz}), 7.21$ (dd, $2 \mathrm{H}, J$ 8.7, $0.6 \mathrm{~Hz}), 7.50$ (bs, 1H), 7.69 (dt, 2H, $J 8.4,1.8 \mathrm{~Hz}) ;{ }^{13} \mathrm{C}$ NMR $(75 \mathrm{MHz}$, $\left.\mathrm{CDCl}_{3}\right) \delta 21.4\left(\mathrm{CH}_{3}\right), 22.1\left(\mathrm{CH}_{2}\right), 26.8\left(\mathrm{CH}_{2}\right), 56.3\left(\mathrm{CH}_{3}\right)$, $57.0\left(\mathrm{CH}_{3}\right), 77.3(\mathrm{CH}), 77.5(\mathrm{CH}), 117.9(\mathrm{CH}), 120.6(\mathrm{CH})$, $127.1(\mathrm{CH}), 129.5(\mathrm{CH}), 130.5(\mathrm{CH}), 131.1(\mathrm{C}), 136.1(\mathrm{C})$, 136.4 (C), 137.7 (C), 143.7 (C); HRMS [ESI(+)-TOF]: calcd. for $\left[\mathrm{C}_{19} \mathrm{H}_{23} \mathrm{NO}_{4} \mathrm{~S}+\mathrm{Na}\right]^{+}: 384.1245$; found: 384.1248 .

\section{Supplementary Information}

Supplementary Information $\left({ }^{1} \mathrm{H}\right.$ NMR and ${ }^{13} \mathrm{C}$ NMR spectra) is available free of charge at http://jbcs.sbq.org.br as PDF file.

\section{Acknowledgments}

The authors thank CAPES, FAPESP and CNPq for financial support.

\section{References}

1. Lee, J.; Kim, H.; Lee, T. G.; Yang, I.; Won, D. H.; Choi, H.; Nam, S.-J.; Kang, H.; J. Nat. Prod. 2014, 77, 1528.
2. Kim, S.-H.; Kwon, S. H.; Park, S.-H.; Lee, J. K.; Bang, H.-S.; Nam, S.-J.; Kwon, H. C.; Shin, J.; Oh, D.-C.; Org. Lett. 2013, 15, 1834.

3. Ferraz, H. M. C.; Aguilar, A. M.; Silva Jr., L. F.; Craveiro, M. V.; Quim. Nova 2005, 28, 703.

4. Loh, C. C. J.; Atodiresei, I.; Enders, D.; Chem. Eur. J. 2013, 19, 10822.

5. Qian, H.; Zhao, W.; Sung, H. H. Y.; Williams, I. D.; Sun, J.; Chem. Commun. 2013, 49, 4361.

6. Hu, J.; Hirao, H.; Li, Y.; Zhou, J.; Angew. Chem., Int. Ed. 2013, 52,8676 .

7. Li, H.-H.; Zhang, X.; Jin, Y.-H.; Tian, S.-K.; Asian J. Org. Chem. 2013, 2, 290.

8. Nishimura, T.; Nagamoto, M.; Ebe, Y.; Hayashi, T.; Chem. Sci. 2013, 4, 4499.

9. Yamamoto, Y.; Saigoku, T.; Ohgai, T.; Nishiyama, H.; Itoh, K.; Chem. Commun. 2004, 2702.

10. Fananas, F. J.; Alvarez-Perez, M.; Rodriguez, F.; Chem. Eur. J. 2005, 11, 5938.

11. Urdabayev, N. K.; Popik, V. V.; J. Am. Chem. Soc. 2004, 126, 4058.

12. Kammath, V. B.; Solomek, T.; Ngoy, B. P.; Heger, D.; Klan, P.; Rubina, M.; Givens, R. S.; J. Org. Chem. 2013, 78, 1718.

13. Siqueira, F. A.; Ishikawa, E. E.; Fogaca, A.; Faccio, A. T.; Carneiro, V. M. T.; Soares, R. R. S.; Utaka, A.; Tebeka, I. R. M.; Bielawski, M.; Olofsson, B.; Silva Jr., L. F.; J. Braz. Chem. Soc. 2011, 22, 1795.

14. Gross, M. F.; Beaudoin, S.; McNaughton-Smith, G.; Amato, G. S.; Castle, N. A.; Huang, C.; Zou, A.; Yu, W.; Bioorg. Med. Chem. Lett. 2007, 17, 2849.

15. Kang, I.-J.; Wang, L.-W.; Yeh, T.-K.; Lee, C.-C.; Lee, Y.-C.; Hsu, S.-J.; Wu, Y.-S.; Wang, J.-C.; Chao, Y.-S.; Yueh, A.; Chern, J.-H.; Bioorg. Med. Chem. 2010, 18, 6414.

16. Li, H.; Ren, X.; Leblanc, E.; Frewin, K.; Rennie, P. S.; Cherkasov, A.; J. Chem. Inf. Model. 2013, 53, 123.

17. Furse, K. E.; Lybrand, T. P.; J. Med. Chem. 2003, 46, 4450.

18. Jagtap, P. G.; Baloglu, E.; Southan, G. J.; Mabley, J. G.; Li, H. S.; Zhou, J.; van Duzer, J.; Salzman, A. L.; Szabo, C.; J. Med. Chem. 2005, 48, 5100 .

19. Jin, Q.; Han, X. H.; Hong, S. S.; Lee, C.; Choe, S.; Lee, D.; Kim, Y.; Hong, J. T.; Lee, M. K.; Hwang, B. Y.; Bioorg. Med. Chem. Lett. 2012, 22, 973.

20. McLean, T. H.; Chambers, J. J.; Parrish, J. C.; Braden, M. R.; Marona-Lewicka, D.; Kurrasch-Orbaugh, D.; Nichols, D. E.; J. Med. Chem. 2006, 49, 4269.

21. Fukatsu, K.; Uchikawa, O.; Kawada, M.; Yamano, T.; Yamashita, M.; Kato, K.; Hirai, K.; Hinuma, S.; Miyamoto, M.; Ohkawa, S.; J. Med. Chem. 2002, 45, 4212.

22. Sheng, R.; Lin, X.; Li, J. Y.; Jiang, Y. K.; Shang, Z. C.; Hu, Y. Z.; Bioorg. Med. Chem. Lett. 2005, 15, 3834. 
23. Wirth, T.; Hypervalent Iodine Chemistry: Modern Developments in Organic Synthesis; Springer: Berlin, Germany, 2003.

24. Zhdankin, V. V.; Hypervalent Iodine Chemistry: Preparation, Structure, and Synthetic Applications of Polyvalent Iodine Compounds; Wiley: Hoboken, New Jersey, US, 2013.

25. Zhdankin, V. V.; Stang, P. J.; Chem. Rev. 2008, 108, 5299.

26. Silva Jr., L. F.; Olofsson, B.; Nat. Prod. Rep. 2011, 28, 1722.

27. Phipps, R. J.; Gaunt, M. J.; Science 2009, 323, 1593.

28. Ochiai, M.; Miyamoto, K.; Kaneaki, T.; Hayashi, S.; Nakanishi, W.; Science 2011, 332, 448.

29. Silva Jr., L. F.; Siqueira, F. A.; Pedrozo, E. C.; Vieira, F. Y. M.; Doriguetto, A. C.; Org. Lett. 2007, 9, 1433.

30. Bianco, G. G.; Ferraz, H. M. C.; Costa, A. M.; Costa-Lotufo, L. V.; Pessoa, C.; de Moraes, M. O.; Schrems, M. G.; Pfaltz, A.; Silva Jr., L. F.; J. Org. Chem. 2009, 74, 2561.

31. Tebeka, I. R. M.; Longato, G. B.; Craveiro, M. V.; de Carvalho, J. E.; Ruiz, A. L. T. G.; Silva Jr., L. F.; Chem. Eur. J. 2012, 18, 16890 .

32. Schneider, T. L.; Halloran, K. T.; Hillner, J. A.; Conry, R. R.; Linton, B. R.; Chem. Eur. J. 2013, 19, 15101.

33. Kilpatrick, B.; Heller, M.; Arns, S.; Chem. Commun. 2013, 49, 514.

34. Sultane, P. R.; Mete, T. B.; Bhat, R. G.; Org. Biomol. Chem. 2014, 12, 261.

35. Silva, L. F.; Sousa, R. M. F.; Ferraz, H. M. C.; Aguilar, A. M.; J. Braz. Chem. Soc. 2005, 16, 1160.

36. Phipps, R. J.; McMurray, L.; Ritter, S.; Duong, H. A.; Gaunt, M. J.; J. Am. Chem. Soc. 2012, 134, 10773.
37. Trenner, J.; Depken, C.; Weber, T.; Breder, A.; Angew. Chem., Int. Ed. 2013, 52, 8952.

38. Hohenlohe-Oehringen, K.; Monatsh. Chem. 1958, 89, 429.

39. Gemma, S.; Butini, S.; Fattorusso, C.; Fiorini, I.; Nacci, V.; Bellebaum, K.; McKissic, D.; Saxena, A.; Campiani, G.; Tetrahedron 2003, 59, 87.

40. Reddy, K. R. K. K.; Longato, G. B.; de Carvalho, J. E.; Ruiz, A. L. T. G.; Silva Jr., L. F.; Molecules 2012, 17, 9621.

41. Kita, Y.; Tohma, H.; Hatanaka, K.; Takada, T.; Fujita, S.; Mitoh, S.; Sakurai, H.; Oka, S.; J. Am. Chem. Soc. 1994, 116, 3684.

42. Dohi, T.; Ito, M.; Yamaoka, N.; Morimoto, K.; Fujioka, H.; Kita, Y.; Tetrahedron 2009, 65, 10797.

43. Williams, D. B. G.; Lawton, M.; J. Org. Chem. 2010, 75, 8351.

44. Rufer, C.; Kessler, H. J.; Schroeder, E.; Damerius, A.; Chim. Ther. 1973, 8, 567.

45. Pappo, R.; US pat. 33189071967.

46. Eastham, J. F.; Larkin, D. R.; J. Am. Chem. Soc. 1958, 80, 2887.

47. Jimenez-Teja, D.; Daoubi, M.; Collado, I. G.; Hernandez-Galan, R.; Tetrahedron 2009, 65, 3392.

48. Aaseng, J. E.; Melnes, S.; Reian, G.; Gautun, O. R.; Tetrahedron 2010, 66, 9790.

Submitted: January 8, 2016

Published online: March 1, 2016

FAPESP has sponsored the publication of this article. 